\section{Accepted Manuscript}

Impulsive observer design for a class of switched nonlinear systems with unknown inputs

Tao Zhan, Shuping Ma, Xinzhi Liu, Hao Chen

PII:

DOI:

$$
\text { S0016-0032(19)30407-7 }
$$

Reference:

$$
\text { FI } 3978
$$

To appear in:

Journal of the Franklin Institute

$\begin{array}{ll}\text { Received date: } & \text { 7 January } 2019 \\ \text { Revised date: } & \text { 1 May } 2019 \\ \text { Accepted date: } & \text { 28 May } 2019\end{array}$

$\begin{array}{ll}\text { Received date: } & \text { 7 January } 2019 \\ \text { Revised date: } & \text { 1 May } 2019 \\ \text { Accepted date: } & \text { 28 May } 2019\end{array}$

$\begin{array}{ll}\text { Received date: } & \text { 7 January } 2019 \\ \text { Revised date: } & \text { 1 May } 2019 \\ \text { Accepted date: } & \text { 28 May } 2019\end{array}$

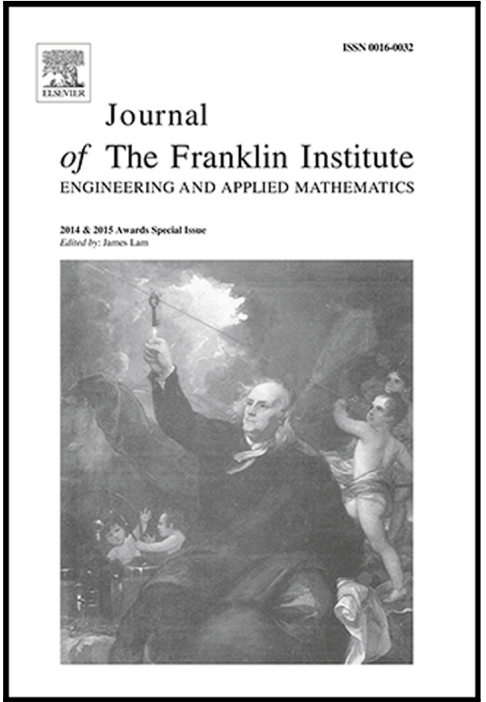

Please cite this article as: Tao Zhan, Shuping Ma, Xinzhi Liu, Hao Chen, Impulsive observer design for a class of switched nonlinear systems with unknown inputs, Journal of the Franklin Institute (2019), doi: https://doi.org/10.1016/j.jfranklin.2019.05.039

This is a PDF file of an unedited manuscript that has been accepted for publication. As a service to our customers we are providing this early version of the manuscript. The manuscript will undergo copyediting, typesetting, and review of the resulting proof before it is published in its final form. Please note that during the production process errors may be discovered which could affect the content, and all legal disclaimers that apply to the journal pertain.

The final publication is available at Elsevier via https://doi.org/10.1016/j.jfranklin.2019.05.039. (C) 2019. This manuscript version is made available under the CC-BY-NC-ND 4.0 license http://creativecommons.org/licenses/by-nc-nd/4.0/ 


\title{
Impulsive observer design for a class of switched nonlinear systems with unknown inputs 放
}

\author{
Tao Zhan ${ }^{\mathrm{a}}$, Shuping Ma ${ }^{\mathrm{a}, *}$, Xinzhi Liu ${ }^{\mathrm{b}}$, Hao Chen ${ }^{\mathrm{b}, \mathrm{c}}$ \\ a School of Mathematics, Shandong University, Jinan, Shandong 250100, China \\ ${ }^{b}$ Department of Applied Mathematics, University of Waterloo, Waterloo, Ontario, N2L $3 G 1$ Canada \\ ${ }^{c}$ College of Electrical and Information Engineering, Southwest Minzu University, Chengdu, Sichuan 610041, China
}

\begin{abstract}
This paper investigates hybrid observer design of a class of unknown input switched nonlinear systems. The distinguishing feature of the proposed method is that the stability of all subsystems of the error switched systems is not necessarily required. First, an output derivative-based method and time-varying coordinate transformation are considered to eliminate the unknown input. Then in order to maintain a satisfactory estimation performance, an impulsive full-order and switched reduced-order observer are developed with a pair of upper and lower dwell time bounds and constructing time-varying Lyapunov functions combined with convex combination technique. In addition, the time-varying Lyapunov functions method is also used to analyze the stability of a class of error switched nonlinear systems with stable subsystems. Finally, two examples are presented to demonstrate the effectiveness of the proposed method.
\end{abstract} Keywords: Impulsive full-order observer, nonlinear switched systems, reduced-order switched observer, unknown inputs.

\section{Introduction}

Switched systems belong to an important class of hybrid systems, which consist of a family of subsystems described by continuous or discrete-time dynamics. Such systems have gained considerable interests in both theoretical researches and practical applications, such as power electronics, chaos generators, traffic control and intercepting missiles [1]. In the last two decades, some fundamental theories of switched systems such as controllability, stability and stabilization have been established, see [2-5] and references therein. Meanwhile, great efforts have also been put on the state estimations or observer designs for switched systems [6-9], since full or partial information of state is unavailable in abundant practical applications. However, these switched systems do not contain unknown inputs. As is known, there are many situations in practical systems where disturbances or partial inputs are inaccessible, such as fault detection and isolation [10], chaotic secure communication [11] and the cutting forces exerted in machine tools [12], which can all be viewed as unknown inputs. Therefore, this paper will focus on state estimation of switched systems with unknown inputs.

\footnotetext{
This work is supported by National Natural Science Foundation of China (61473173,61703353), Major International (Regional) Joint Research Project of the National Natural Science Foundation of China (NSFC)(61320106011), Natural Sciences and Engineering Research Council of Canada (NSERC).

${ }^{*}$ Corresponding author

Email address: mashup@sdu.edu.cn (Shuping Ma)
} 
Recently, many interesting results on unknown inputs observer design for switched systems have been reported in literature [13-16]. We note that all of the above results are based on the assumption that the output of the system under consideration is measured continuously. However, the outputs of many control systems are only available at discrete sampled instants because of the applications of digital sensors. In this case, proposing an impulsive observer is needed, which can not only be updated in an impulsive fashion, but also save the bandwidth of networks and communication cost when the measured information from the system transport to the observer at discrete time instants in comparison with continuous-time observers. Then, a impulsive error dynamic system is constructed, which has been investigated extensively [17-19]. In [20], a full-order impulsive observer was designed firstly for nonlinear continuous-time systems. Then some different impulsive observers, such as adaptive impulsive observers and impulsive functional observers, were proposed for different systems in [21-24]. But for switched nonlinear systems, only [25] investigated the adaptive impulsive observers, seldom did scholars consider this issue for switched systems with unknown inputs.

In addition, on the approach of decoupling unknown inputs from the dynandics of unavailable state component, the observer design for the unknown input switched systems are studied in [26-29]. In [26], it should be pointed out that the convergence condition of the error dynamics was based on the existence of a common time-invariant Lyapunov function. As we know, it is not easy to find this common timeinvariant Lyapunov function, and many switched systems can achieve asymptotic stability under some proper switching law without possessing a common time-invariant Lyapunov function. Then how to design an appropriate switching law became one of the most challenging problems in studying switched systems. Further, we find that all of results in $[21,25-29]$ have the requirements that all or parts of error subsystems are stable. Thus, questions naturally arise: is it possible, based on the dwell-time technique, to achieve the observers design of switched nonlinear systems with unknown inputs without posing any stability requirements on error subsystems of switched systems? If possible, under what conditions can we achieve this goal and how?

To study the problems proposed above, different approaches of decoupling unknown inputs are applied firstly to design the hybrid observer for switched nonlinear systems with all unstable error subsystems. The main characteristics of the paper are summarized as follows.

1). Compared with the existing literature, the impulsive full-order observer is designed even if each error subsystem is unstable. Furthermore, a novel algorithm is given to avoid the limitation of the linearization method.

2). Different from [26-28], when there is no the assumption that the original switched subsystems are strong detectable, the reduced-order observer can still be designed successfully.

3). By virtue of time-varying Lyapunov function that sufficiently captures the hybrid characteristic of switched impulsive systems, sufficient conditions for ensuring the stability of the error switched systems are constructed in the framework of the convex combination technique.

This paper is organized as follows. In section 2, system description and necessary preliminaries are given. The main results are presented in Section 3 . An illustrative example along with numerical and simulation results is provided in Section 4. 


\section{Preliminaries}

We shall introduce, in this section, some notations, definitions and lemmas, which will be used in the later sections.

Let $X \geq 0$ (or, $X>0$ ) denote the symmetric matrix $X$ that is semi-positive definite (or, positive definite). $I$ and 0 represent, respectively, the identity matrix and zero matrix with appropriate dimensions. $\mathbb{R}$ and $\mathbb{R}^{+}$stand for the real numbers set and positive real numbers set. $\mathbb{R}^{n}$ and $\mathbb{R}^{m \times n}$ denote, respectively, the $n$-dimensional Euclidean space, and the set of all $m \times n$ real matrices. For any matrix $X, X^{T}$ denotes its transpose, $X^{-1}$ means its inverse, $X^{+}$is the moore-Penrose inverse. If $X$ has full column rank, then $X^{+}=\left(X^{T} X\right)^{-1} X^{T}$; if $X$ has full row rank, then $X^{+}=X^{T}\left(X X^{T}\right)^{-1}$. The superscript ' $T$ ' denotes the transpose of a matrix, the symbol $*$ is the transposed elements in the symmetric position of a matrix, $H e(X)$ represents $X+X^{T}$. eig $(X)$ represents the eigenvalues of matrix $X$.

Consider a class of nonlinear switched systems with unknown inputs described by

$$
\left\{\begin{array}{l}
\dot{x}(t)=A_{\sigma(t)} x(t)+B_{\sigma(t)} u(t)+D_{\sigma(t)} w(t)+f_{\sigma(t)}(t, x(t)), \\
y(t)=C_{\sigma(t)} x(t),
\end{array}\right.
$$

where $x(t) \in \mathbb{R}^{n}$ is state, $u(t) \in \mathbb{R}^{m}$ is control input, $w(t) \in \mathbb{R}^{p}$ is unknown input vectors, and $y(t) \in \mathbb{R}^{q}$ is measurement output. $f_{\sigma(t)}(t, x(t)):[0, \infty) \times \mathbb{R}^{n} \rightarrow \mathbb{R}^{n}$ are continuous functions in their arguments. The matrices $A_{\sigma(t)}, B_{\sigma(t)}, C_{\sigma(t)}$ and $D_{\sigma(t)}$ are constant matrices with appropriate dimensions. $x\left(t_{k}^{+}\right)=$ $\lim _{h \rightarrow 0^{+}} x\left(t_{k}+h\right)$ and $x\left(t_{k}^{-}\right)=\lim _{h \rightarrow 0^{-}} x\left(t_{k}-h\right) . \sigma(t):\left[t_{0}, \infty\right) \rightarrow M=\{1,2, \ldots, m\}$ is the switching signal, where $m$ is a positive integer. $j \in M$ is the active mode at time $t$ if $\sigma(t)=j \in M$ for $t \in\left[t_{k}, t_{k+1}\right)$ with $k=0,1,2, \ldots$, where $t_{k}$ is the $k$ th switching time instant. The time sequence $\left\{t_{k}, k=0,1,2, \ldots\right\}$ belongs to $\Delta\left(\delta_{1}, \delta_{2}\right) \triangleq\left\{t_{0}<t_{1}<\ldots<t_{k}<\ldots, \lim _{k \rightarrow+\infty} t_{k}=+\infty, \delta_{1} \leq t_{k+1}-t_{k} \leq \delta_{2}\right\}$, where $\delta_{1}$ and $\delta_{2}$ are minimum and maximum dwell times, respectively. The purpose of this paper is to develop a full-order impulsive observer and a reduced-order switched observer for the switched nonlinear system (1) under the following assumptions.

Assumption 1 For any $i \in M$, there exist matrices $F_{i} \in \mathbb{R}^{n \times n}$ such that,

$$
\left\|f_{i}\left(t, x_{1}\right)-f_{i}\left(t, x_{2}\right)\right\| \leq\left\|F_{i}\left(x_{1}-x_{2}\right)\right\| .
$$

Assumption $2 \operatorname{rank}\left(C_{i} D_{i}\right)=\operatorname{rank}\left(D_{i}\right)=p, \forall i \in M$.

\section{Main results}

3.1. Full-order impulsive observer

In this section, for observer design of system (1), it is necessary to eliminate the unknown input influences. Under Assumption 2, there exist matrices $E_{i} \in \mathbb{R}^{n \times q}$ such that $E_{i} C_{i} D_{i}=D_{i}$ for any $i \in M$. Then the general solution of $E_{i}$ is given by

$$
E_{i}=D_{i}\left(C_{i} D_{i}\right)^{+}+G_{i}\left(I-\left(C_{i} D_{i}\right)\left(C_{i} D_{i}\right)^{+}\right)
$$

where $G_{i}$ is an arbitrary matrix with appropriate dimensions. Thus, we have

$$
\begin{aligned}
D_{i} w(t)=E_{i} C_{i} D_{i} w(t) & =E_{i} C_{i}\left(\dot{x}(t)-A_{i} x(t)-B_{i} u(t)-f_{i}(t, x(t))\right) \\
& =E_{i} \dot{y}(t)-E_{i} C_{i} A_{i} x(t)-E_{i} C_{i} B_{i} u(t)-E_{i} C_{i} f_{i}(t, x(t)) .
\end{aligned}
$$


Substituting (3) into (1) yields

$$
\left.\dot{x}(t)=N_{i} A_{i} x(t)+N_{i} B_{i} u(t)+N_{i} f_{i}(t, x(t))\right)+E_{i} \dot{y}(t),
$$

where $N_{i}=I-E_{i} C_{i}$.

Remark 3.1. In fact, for system (4), a general full-order observer is designed as

$$
\left.\dot{\hat{x}}(t)=N_{i} A_{i} \hat{x}(t)+N_{i} B_{i} u(t)+N_{i} f_{i}(t, \hat{x}(t))\right)+E_{i} \dot{y}(t)+L_{i}(y(t)-\hat{y}(t)),
$$

where $\hat{x}(t)$ is the estimation of $x(t), \hat{y}(t)=C \hat{x}(t)$ is the corresponding output. $L_{i}$ are observer gain matrices. Obviously, the derivative of $y(t)$ is contained, which is difficult to measure in practical applications and may cause high frequency noise in observer design. In order to avoid this problem, a coordinate transformation is introduced, which may lead the estimated state jump in switched systems, thus an impulsive observer is needed.

The impulsive observer for switched system (1) has the form:

$$
\left\{\begin{array}{l}
\left.\dot{z}(t)=N_{i} A_{i} \hat{x}(t)+N_{i} B_{i} u(t)+N_{i} f_{i}(t, \hat{x}(t))\right), t \neq t_{k} \\
z\left(t_{k}^{+}\right)=z\left(t_{k}^{-}\right)+L_{i}\left(y\left(t_{k}^{-}\right)-\hat{y}\left(t_{k}^{-}\right)\right), t=t_{k} \\
\hat{x}(t)=z(t)+E_{i} y(t)
\end{array}\right.
$$

where $z$ is the observer state, $\hat{x}$ is the estimation of $x, L_{i}$ are the observer gain matrices to be designed.

In addition, it is assumed that $z\left(t_{k}\right)=z\left(t_{k}^{+}\right)$, i.e., the solutions of the impulsive observer are right continuous, and then $\hat{x}\left(t_{k}\right)=\hat{x}\left(t_{k}^{+}\right)$.

Define $e=x-\hat{x}$. From (1) and (5), the estimation error dynamic of subsystem $i \in M$ is

$$
\left\{\begin{array}{l}
\dot{e}(t)=N_{i} A_{i} e(t)+N_{i} \Delta f_{i}(t, x, \hat{x}), t \neq t_{k}, \\
e\left(t_{k}\right)=\left(I-L_{i} C_{i}\right) e\left(t_{k}^{-}\right), t=t_{k}
\end{array}\right.
$$

where $\Delta f_{i}(t, x, \hat{x})=f_{i}(t, x(t))-f_{i}(t, \hat{x}(t))$.

By virtue of Assumption 1, one has

$$
\left\|\Delta f_{i}(t, x, \hat{x})\right\| \leq\left\|F_{i} e\right\|, \forall i \in M \text {. }
$$

Remark 3.2. In [29], a full-order impulsive reset observer is built with the premise that each error subsystem is stable. Compared with this situation, it is more challenging and difficult that none of the individual error) subsystems is stable. Thus, our task in the next section is to identify certain classes of switching signals satisfying (10) and in the meantime to design observer gains to ensure stability of the switched error system (6).

Case a: all error subsystems are unstable.

Theorem 3.1. Consider switched system (1) satisfying Assumptions 1-2 and the time sequence $t_{k} \in$ $\Delta\left(\delta_{1}, \delta_{2}\right)$. For given $\eta>0,0<\mu<1$ and $\rho_{i}>1$, if there exist $P_{i 1}>0, P_{i 2}>0, G_{i} \in \mathbb{R}^{n \times q}, \bar{L}_{i} \in \mathbb{R}^{n \times q}$, $\epsilon_{i m n}>0, i, j \in M, i \neq j, m, n=1,2$, such that

$$
\left[\begin{array}{cc}
\Omega_{i m n} & P_{i m} T_{i}-P_{i m} G_{i} H_{i} C_{i} \\
* & -\epsilon_{i m n} I
\end{array}\right]<0
$$




$$
\begin{aligned}
& {\left[\begin{array}{cc}
-\mu P_{j 1} & P_{i 2}-C_{i}^{T} \bar{L}_{i}^{T} \\
* & -\rho_{i} P_{i 2}
\end{array}\right]<0,} \\
& \ln \mu+\eta \delta_{2}<0
\end{aligned}
$$

where

$\Omega_{i m n}=\theta_{i} P_{i m}+H e\left\{P_{i m}\left(T_{i}-G_{i} H_{i} C_{i}\right) A_{i}\right\}+\frac{1}{\delta_{n}}\left(P_{i 1}-P_{i 2}\right)-\eta P_{i m}+\epsilon_{i m n} F_{i}^{T} F_{i}$ with $\theta_{i}=\frac{\ln \rho_{i}}{\delta_{1}}$, $T_{i}=I-D_{i}\left(C_{i} D_{i}\right)^{+} C_{i}, H_{i}=I-C_{i} D_{i}\left(C_{i} D_{i}\right)^{+}$,

then the switched observer error dynamic system (6) is asymptotically stable. The observer gains are given by

$$
L_{i}=P_{i 2}^{-1} \bar{L}_{i}, i \in M
$$

Proof: For $\forall t \in\left[t_{k}, t_{k+1}\right)$, define $\delta_{k}=t_{k+1}-t_{k}, \kappa(t)=\frac{t-t_{k}}{\delta_{k}}, \tilde{\kappa}(t)=1-\kappa(t)$, then it follows that $\kappa(t) \in[0,1], \kappa\left(t_{k}\right)=0, \kappa\left(t_{k}^{-}\right)=1$.

Suppose $\sigma(t)=i, t \in\left[t_{k}, t_{k+1}\right)$. Let $e(t)$ be a solution of system (6) and

$$
V_{i}(e)=\psi(t) e^{T} P_{i}(t) e, i \in M,
$$

where $\psi(t)=\rho_{i}^{\kappa(t)-1}, P_{i}(t)=\kappa(t) P_{i 1}+\tilde{\kappa}(t) P_{i 2}$. Define $V_{i}(t) \leqslant V_{i}(e(t))$, then taking derivative of $V_{i}(t)$ along the trajectory of system (6) yields

$$
\begin{aligned}
\dot{V}_{i}(t)-\eta V_{i}(t)= & \dot{\psi}(t) e^{T} P_{i}(t) e+2 \psi(t) e^{T} P_{i}(t) \dot{e}+\psi(t) e^{T} \dot{P}_{i}(t) e-\eta \psi(t) e^{T} P_{i}(t) e \\
= & \psi(t)\left\{e^{T}\left[\frac{\ln \rho_{i}}{\delta_{k}} P_{i}(t)+2 P_{i}(t) N_{i} A_{i}-\frac{1}{\delta_{k}}\left(P_{i 1}-P_{i 2}\right)-\eta P_{i}(t)\right] e\right. \\
& \left.+2 e^{T} P_{i}(t) N_{i} \Delta f_{i}\right\} \\
\leq & \psi(t)\left\{e^{T}\left[\theta_{i} P_{i}(t)+2 P_{i}(t) N_{i} A_{i}+\frac{1}{\delta_{k}}\left(P_{i 1}-P_{i 2}\right)-\eta P_{i}(t)\right] e+2 e^{T} P_{i}(t) N_{i} \Delta f_{i}\right\},
\end{aligned}
$$

where $\Delta f_{i}=\Delta f_{i}(t, x, \hat{x}), \theta_{i}=\frac{\ln \rho_{\uparrow}}{\delta_{1}}$.

We choose a function $\nu(t)=\left(\frac{1}{\delta_{k}}-\frac{1}{\delta_{2}}\right) /\left(\frac{1}{\delta_{1}}-\frac{1}{\delta_{2}}\right) \in[0,1]$ and $\tilde{\nu}(t)=1-\nu(t)$, such that

$$
\frac{1}{\delta_{k}}=\frac{1}{\delta_{1}} \nu(t)+\frac{1}{\delta_{2}} \tilde{\nu}(t)
$$

From (12) and (13), one has that

$$
\begin{aligned}
\dot{V}_{i}(t)-\eta V_{i}(t) \leq & \psi(t)\left\{e ^ { T } \left[\theta_{i}\left(\kappa(t) P_{i 1}+\tilde{\kappa}(t) P_{i 2}\right)+2\left(\kappa(t) P_{i 1}+\tilde{\kappa}(t) P_{i 2}\right) N_{i} A_{i}-\eta P_{i}(t)\right.\right. \\
+ & \left.\left.\left(\frac{1}{\delta_{1}} \nu(t)+\frac{1}{\delta_{2}} \tilde{\nu}(t)\right)\left(P_{i 1}-P_{i 2}\right)\right] e+2 e^{T}\left(\kappa(t) P_{i 1}+\tilde{\kappa}(t) P_{i 2}\right) N_{i} \Delta f_{i}\right\} \\
= & \psi(t)\left\{e ^ { T } \left[\kappa(t)\left(\theta_{i} P_{i 1}+2 P_{i 1} N_{i} A_{i}\right)+\tilde{\kappa}(t)\left(\theta_{i} P_{i 2}+2 P_{i 2} N_{i} A_{i}\right)-\eta P_{i}(t)\right.\right. \\
& \left.\left.+\left(\frac{1}{\delta_{1}} \nu(t)+\frac{1}{\delta_{2}} \tilde{\nu}(t)\right)\left(P_{i 1}-P_{i 2}\right)\right] e+2 e^{T}\left(\kappa(t) P_{i 1}+\tilde{\kappa}(t) P_{i 2}\right) N_{i} \Delta f_{i}\right\} \\
= & \psi(t) e^{T}\left\{\kappa ( t ) \left[\nu(t)\left(\theta_{i} P_{i 1}+2 P_{i 1} N_{i} A_{i}+\frac{1}{\delta_{1}}\left(P_{i 1}-P_{i 2}\right)-\eta P_{i 1}\right)\right.\right. \\
& \left.+\tilde{\nu}(t)\left(\theta_{i} P_{i 1}+2 P_{i 1} N_{i} A_{i}+\frac{1}{\delta_{2}}\left(P_{i 1}-P_{i 2}\right)-\eta P_{i 1}\right)\right] \\
& +\tilde{\kappa}(t)\left[\nu(t)\left(\theta_{i} P_{i 2}+2 P_{i 2} N_{i} A_{i}+\frac{1}{\delta_{1}}\left(P_{i 1}-P_{i 2}\right)-\eta P_{i 2}\right)\right. \\
& \left.\left.+\tilde{\nu}(t)\left(\theta_{i} P_{i 2}+2 P_{i 2} N_{i} A_{i}+\frac{1}{\delta_{2}}\left(P_{i 1}-P_{i 2}\right)-\eta P_{i 2}\right)\right]\right\} e \\
& +2 \psi(t) e^{T}\left(\kappa(t) P_{i 1}+\tilde{\kappa}(t) P_{i 2}\right) N_{i} \Delta f_{i} .
\end{aligned}
$$


According to (7), there exist $\epsilon_{i}(t)=\kappa(t) \nu(t) \epsilon_{i 11}+\kappa(t) \tilde{\nu}(t) \epsilon_{i 12}+\tilde{\kappa}(t) \nu(t) \epsilon_{i 21}+\tilde{\kappa}(t) \tilde{\nu}(t) \epsilon_{i 22}$ such that

$$
\epsilon_{i}(t)\left(e^{T} F_{i}^{T} F_{i} e-\Delta f_{i}^{T} \Delta f_{i}\right) \geq 0 .
$$

Combining (14) and (15), we have

$$
\begin{aligned}
\dot{V}_{i}(t)-\eta V_{i}(t) \leq & \psi(t) e^{T}\left\{\kappa ( t ) \left[\nu(t)\left(\theta_{i} P_{i 1}+2 P_{i 1} N_{i} A_{i}+\frac{1}{\delta_{1}}\left(P_{i 1}-P_{i 2}\right)-\eta P_{i 1}\right)\right.\right. \\
& \left.+\tilde{\nu}(t)\left(\theta_{i} P_{i 1}+2 P_{i 1} N_{i} A_{i}+\frac{1}{\delta_{2}}\left(P_{i 1}-P_{i 2}\right)-\eta P_{i 1}\right)\right] \\
& +\tilde{\kappa}(t)\left[\nu(t)\left(\theta_{i} P_{i 2}+2 P_{i 2} N_{i} A_{i}+\frac{1}{\delta_{1}}\left(P_{i 1}-P_{i 2}\right)-\eta P_{i 2}\right)\right. \\
& \left.\left.+\tilde{\nu}(t)\left(\theta_{i} P_{i 2}+2 P_{i 2} N_{i} A_{i}+\frac{1}{\delta_{2}}\left(P_{i 1}-P_{i 2}\right)-\eta P_{i 2}\right)\right]\right\} e \\
& +2 \psi(t) e^{T}\left(\kappa(t) P_{i 1}+\tilde{\kappa}(t) P_{i 2}\right) N_{i} \Delta f_{i}+\epsilon_{i}(t)\left(e^{T} F_{i}^{T} F_{i} e-\Delta f_{i}^{T} \Delta f_{i}\right) \\
= & \psi(t)\left\{\kappa(t) \nu(t) \Phi_{i}^{11}+\kappa(t) \tilde{\nu}(t) \Phi_{i}^{12}+\tilde{\kappa}(t) \nu(t) \Phi_{i}^{21}+\tilde{\kappa}(t) \tilde{\nu}(t) \Phi_{i}^{22}\right\}
\end{aligned}
$$

where

$$
\begin{aligned}
\Phi_{i}^{m n}= & e^{T}\left[\theta_{i} P_{i m}+P_{i m} N_{i} A_{i}+A_{i}^{T} N_{i}^{T} P_{i m}+\frac{1}{\delta_{n}}\left(P_{i 1}-P_{i 2}\right) \measuredangle \eta P_{i m}+\epsilon_{i m n} F_{i}^{T} F_{i}\right] e \\
& +2 e^{T} P_{i m} N_{i} \Delta f_{i}-\epsilon_{i m n} \Delta f_{i}^{T} \Delta f_{i} \\
= & {\left[\begin{array}{cc}
e^{T} & \Delta f_{i}^{T}
\end{array}\right] \tilde{\Phi}_{i}^{m n}\left[\begin{array}{c}
e \\
\Delta f_{i}
\end{array}\right], }
\end{aligned}
$$

where $\tilde{\Phi}_{i}^{m n}=\left[\begin{array}{cc}\theta_{i} P_{i m}+P_{i m} N_{i} A_{i}+A_{i}{ }^{T} N_{i}^{T} P_{i m}+\frac{1}{\delta_{n}}\left(P_{i 1}-P_{i 2}\right)-\eta P_{i m}+\epsilon_{i m n} F_{i}^{T} F_{i} & P_{i m} N_{i} \\ * & -\epsilon_{i m n} I\end{array}\right]$.

Substituting $N_{i}=I-E_{i} C_{i}=T_{i}-G_{i} H_{i} C_{i}$ into $\tilde{\Phi}_{i}^{m n}$ and in view of Schur Complement Lemma, condition (8) is equivalent to $\tilde{\Phi}_{i}^{m n}<0$. It follows from (13) that

$$
\dot{V}_{i}(t)<\eta V_{i}(t), t \neq t_{k}
$$

which implies

$$
V_{i}(t) \leq V_{i}\left(t_{k}\right) \exp \left(\eta\left(t-t_{k}\right)\right), t \in\left[t_{k}, t_{k+1}\right)
$$

At the impulsive switching time point $t=t_{k}, V_{i}\left(t_{k}\right)=\psi\left(t_{k}\right) e^{T}\left(t_{k}\right) P_{i}\left(t_{k}\right) e\left(t_{k}\right)$. Denote $\sigma(t)=j, t \in$ $\left[t_{k-1}, t_{k}\right)$, from $(9)$, it is obtained that

$$
\begin{aligned}
V_{i}\left(t_{k}\right) & =\psi\left(t_{k}\right) e^{T}\left(t_{k}\right) P_{i}\left(t_{k}\right) e\left(t_{k}\right) \\
& =\rho_{i}^{-1} e^{T}\left(t_{k}^{-}\right)\left(I-L_{i} C_{i}\right)^{T} P_{i 2}\left(I-L_{i} C_{i}\right) e\left(t_{k}^{-}\right) \\
& \leq \mu e^{T}\left(t_{k}^{-}\right) P_{j 1} e\left(t_{k}^{-}\right) \\
& =\mu \psi\left(t_{k}^{-}\right) e^{T}\left(t_{k}^{-}\right) P_{j}\left(t_{k}^{-}\right) e\left(t_{k}^{-}\right) \\
& =\mu V_{j}\left(t_{k}^{-}\right) .
\end{aligned}
$$

Combining (17) with above inequality, we have

$$
V_{i}\left(t_{k}\right) \leq \mu V_{j}\left(t_{k}^{-}\right) \leq \mu V_{j}\left(t_{k-1}\right) \exp \left(\eta\left(t_{k}-t_{k-1}\right)\right) \leq V_{j}\left(t_{k-1}\right) \exp \left(\ln \mu+\eta \delta_{2}\right),
$$


From (10) and (19), it follows that $V_{i}\left(t_{k}\right) \leq V_{j}\left(t_{k-1}\right)$, which implies that $V_{\sigma\left(t_{k}\right)}\left(t_{k}\right), k=1,2, \ldots$ is strictly decreasing.

Using the same deduction as above leads to

$$
V_{\sigma\left(t_{k}\right)}\left(t_{k}\right) \leq \exp \left(k\left(\ln \mu+\eta \delta_{2}\right)\right) V_{\sigma\left(t_{0}\right)}\left(t_{0}\right) \leq \exp \left(k\left(\ln \mu+\eta \delta_{2}\right)\right) \max \left\{\lambda_{\max }\left(P_{i l}\right)\right\}\left\|e_{0}\right\|^{2}, l=1,2,
$$

which implies $\lim _{k \rightarrow+\infty} V_{\sigma\left(t_{k}\right)}\left(t_{k}\right)=0$ under the condition (10). From (17), it follows that $V_{\sigma}(t) \leq$ $V_{\sigma}\left(t_{k}\right) \exp \left(\eta \delta_{2}\right)$ for $t \in\left[t_{k}, t_{k+1}\right)$, i.e., $\lim _{t \rightarrow+\infty} V_{\sigma(t)}(t)=0$. From the description of $V_{i}(t)$, we have $\min \left\{\lambda\left(P_{i l}\right)\right\}\|e(t)\|^{2} \leq \rho_{i} V_{i}(t) \leq \rho_{i} \max \left\{\lambda\left(P_{i l}\right)\right\}\|e(t)\|^{2}$. Then

$$
\lim _{t \rightarrow+\infty}\|e(t)\|^{2} \leq \frac{\max \left\{\rho_{i}\right\}}{\min \left\{\lambda\left(P_{i l}\right)\right\}} V_{i}(t)=0,
$$

where $i \in M$ and $l=1,2, \lambda\left(P_{i l}\right)$ is the eigenvalue of matrix $P_{i l}$. Therefore, system (6) is asymptotically stable.

Case b: all error subsystems are stable.

Theorem 3.2. Consider switched system (1) satisfying Assumptions 1-2 and the time sequence $t_{k} \in$ $\Delta\left(\delta_{1}, \delta_{2}\right)$. For given $\eta>0, \mu>0$ and $\rho_{i}>1$, if there exist $P_{i 1}>0, P_{i 2}>0, G_{i} \in \mathbb{R}^{n \times q}, \bar{L}_{i} \in \mathbb{R}^{n \times q}$, $\epsilon_{i m n}>0, i, j \in M, i \neq j, m, n=1,2$, such that (9) and the following inequalities hold:

$$
\begin{aligned}
& {\left[\begin{array}{cc}
\Omega_{i m n} & P_{i m} T_{i}-P_{i m} G_{i} H_{i} C_{i} \\
* & -\epsilon_{i m n} I
\end{array}\right]<0,} \\
& \ln \mu-\eta \delta_{1}<0,
\end{aligned}
$$

where

$\Omega_{i m n}=\theta_{i} P_{i m}+H e\left\{P_{i m}\left(T_{i}-G_{i} H_{i} C_{i}\right) A_{i}\right\}+\frac{1}{\delta_{n}}\left(P_{i 1}-P_{i 2}\right)+\eta P_{i m}+\epsilon_{i m n} F_{i}^{T} F_{i}$ with $\theta_{i}=\frac{\ln \rho_{i}}{\delta_{1}}$, $T_{i}=I-D_{i}\left(C_{i} D_{i}\right)^{+} C_{i}, H_{i}=I-C_{i} D_{i}\left(C_{i} D_{i}\right)^{+}$,

then the switched observer error dynamic system (6) is asymptotically stable. The observer gains are given by

$$
L_{i}=P_{i 2}^{-1} \bar{L}_{i,}, i \in M
$$

Proof: Aceording to the proof in Theorem 3.1, we have

$$
\begin{aligned}
\dot{V}_{i}(t)+\eta V_{i}(t) \leq & \psi(t)\left\{e^{T}\left[\theta_{i} P_{i}(t)+2 P_{i}(t) N_{i} A_{i}+\frac{1}{\delta_{k}}\left(P_{i 1}-P_{i 2}\right)+\eta P_{i}(t)\right] e+2 e^{T} P_{i}(t) N_{i} \Delta f_{i}\right\} \\
\leq & \psi(t) e^{T}\left\{\kappa ( t ) \left[\nu(t)\left(\theta_{i} P_{i 1}+2 P_{i 1} N_{i} A_{i}+\frac{1}{\delta_{1}}\left(P_{i 1}-P_{i 2}\right)+\eta P_{i 1}\right)\right.\right. \\
& \left.+\tilde{\nu}(t)\left(\theta_{i} P_{i 1}+2 P_{i 1} N_{i} A_{i}+\frac{1}{\delta_{2}}\left(P_{i 1}-P_{i 2}\right)+\eta P_{i 1}\right)\right] \\
& +\tilde{\kappa}(t)\left[\nu(t)\left(\theta_{i} P_{i 2}+2 P_{i 2} N_{i} A_{i}+\frac{1}{\delta_{1}}\left(P_{i 1}-P_{i 2}\right)+\eta P_{i 2}\right)\right. \\
& \left.\left.+\tilde{\nu}(t)\left(\theta_{i} P_{i 2}+2 P_{i 2} N_{i} A_{i}+\frac{1}{\delta_{2}}\left(P_{i 1}-P_{i 2}\right)+\eta P_{i 2}\right)\right]\right\} e \\
& +2 \psi(t) e^{T}\left(\kappa(t) P_{i 1}+\tilde{\kappa}(t) P_{i 2}\right) N_{i} \Delta f_{i}+\epsilon_{i}(t)\left(e^{T} F_{i}^{T} F_{i} e-\Delta f_{i}^{T} \Delta f_{i}\right) \\
= & \psi(t)\left\{\kappa(t) \nu(t) \hat{\Phi}_{i}^{11}+\kappa(t) \tilde{\nu}(t) \hat{\Phi}_{i}^{12}+\tilde{\kappa}(t) \nu(t) \hat{\Phi}_{i}^{21}+\tilde{\kappa}(t) \tilde{\nu}(t) \hat{\Phi}_{i}^{22}\right\},
\end{aligned}
$$


where

$$
\begin{aligned}
\hat{\Phi}_{i}^{m n}= & e^{T}\left[\theta_{i} P_{i m}+P_{i m} N_{i} A_{i}+A_{i}{ }^{T} N_{i}^{T} P_{i m}+\frac{1}{\delta_{n}}\left(P_{i 1}-P_{i 2}\right)+\eta P_{i m}+\epsilon_{i m n} F_{i}{ }^{T} F_{i}\right] e \\
& +2 e^{T} P_{i m} N_{i} \Delta f_{i}-\epsilon_{i m n} \Delta f_{i}^{T} \Delta f_{i} \\
= & {\left[\begin{array}{cc}
e^{T} & \Delta f_{i}^{T}
\end{array}\right] \check{\Phi}_{i}^{m n}\left[\begin{array}{c}
e \\
\Delta f_{i}
\end{array}\right], }
\end{aligned}
$$

where $\check{\Phi}_{i}^{m n}=\left[\begin{array}{cc}\theta_{i} P_{i m}+P_{i m} N_{i} A_{i}+A_{i}^{T} N_{i}^{T} P_{i m}+\frac{1}{\delta_{n}}\left(P_{i 1}-P_{i 2}\right)+\eta P_{i m}+\epsilon_{i m n} F_{i}^{T} F_{i} & P_{i m} N_{i} \\ * & -\epsilon_{i m n} T\end{array}\right]$.

From condition (21), it is obtained that

$$
\dot{V}_{i}(t)<-\eta V_{i}(t), t \neq t_{k}
$$

which implies

$$
V_{i}(t) \leq V_{i}\left(t_{k}\right) \exp \left(-\eta\left(t-t_{k}\right)\right), t \in\left[t_{k}, t_{k+1}\right)
$$

At the impulsive switching time point $t=t_{k}$, based on the condition (22), we have $V_{i}\left(t_{k}\right) \leq \mu V_{j}\left(t_{k}^{-}\right)$. Then it implies that

$$
V_{i}\left(t_{k}\right) \leq \mu V_{j}\left(t_{k-1}\right) \exp \left(-\eta\left(t_{k}-t_{k-1}\right)\right) \leq V_{j}\left(t_{k-1}\right) \exp \left(\ln \mu-\eta \delta_{1}\right) .
$$

Combining with (22), it follows that $V_{i}\left(t_{k}\right) \leq V_{j}\left(t_{k-1}\right)$, and then $V_{\sigma\left(t_{k}\right)}\left(t_{k}\right), k=1,2, \ldots$ is strictly decreasing. Similar to the proof in Theorem 3.1, system (6), is asymptotically stable.

In order to solve the nonlinear matrix inequalities in Theorems 3.1 and 3.2, motivated by the linear programming technique, a new type of algorithm is presented as follows.

\section{Algorithm:}

Step 1) Select the appropriate scalars $\mu$ and $\eta$ satisfying $(10)((22))$, by using cvx toolbox in Matlab, equalities (9) can be solved with $P_{i 1}, P_{i 2}>0, i \in M$.

Step 2) Substituting the obtained $P_{i 1}, P_{i 2}$ into $(8)((21))$, then one feasible solution can be calculated as $G_{i}$. Otherwise, go back to step 1 .

Remark 3.3. In [30, 31], the linearization method is often used to deal with the term $P_{i m} G_{i}$. If we also take this method in Theorem 3.1, the corresponding result is given as follows:

Consider switched system (1) satisfying Assumptions 1-2 and the time sequence $t_{k} \in \Delta\left(\delta_{1}, \delta_{2}\right)$. For given $\eta>0,0<\mu<1, \rho_{i}>1$ and constants $k_{1}, k_{2}>0$, if there exist $P_{i}>0, \bar{G}_{i} \in \mathbb{R}^{n \times q}, \bar{L}_{i} \in \mathbb{R}^{n \times q}$, $\epsilon_{i m n} \gg 0, i, j \in M, i \neq j, m, n=1,2$, such that

$$
\left[\begin{array}{cc}
\Omega_{i m n} & k_{m} P_{i} T_{i}-k_{m} P_{i} G_{i} H_{i} C_{i} \\
* & -\epsilon_{i m n} I
\end{array}\right]<0
$$

$$
\left[\begin{array}{cc}
-\mu k_{1} P_{j} & k_{2} P_{i}-C_{i}^{T} \bar{L}_{i}^{T} \\
* & -\rho_{i} k_{2} P_{i}
\end{array}\right]<0
$$

$\ln u+\eta \delta_{2}<0$, 
where

$\Omega_{i m n}=\theta_{i} k_{m} P_{i}+\operatorname{He}\left\{k_{m} P_{i}\left(T_{i}-G_{i} H_{i} C_{i}\right) A_{i}\right\}+\frac{k_{1}-k_{2}}{\delta_{n}} P_{i}-\eta k_{m} P_{i}+\epsilon_{i m n} F_{i}^{T} F_{i}$ with $\theta_{i}=\frac{\ln \rho_{i}}{\delta_{1}}$,

then the switched observer error dynamic system (6) is asymptotically stable. The observer gains are given by $L_{i}=P_{i}^{-1} \bar{L}_{i}, i \in M$ and the matrix $G_{i}=P_{i}^{-1} \bar{G}_{i}$.

Therefore, above result is a special case of Theorem 3.1, then Theorem 3.1 with the algorithm in Remark 3.3 is less conservatism.

\subsection{Reduced-order switched observer}

Under Assumption 2, for any $i \in M$, we introduce the time-varying state transformation $[16]$ :

$$
K_{i}=\left[\begin{array}{c}
D_{i}^{\perp} \\
\left(C_{i} D_{i}\right)^{+} C_{i}
\end{array}\right] \triangleq\left[\begin{array}{c}
D_{i}^{\perp} \\
\check{K}_{i}
\end{array}\right], U_{i}=\left[\begin{array}{c}
\left(C_{i} D_{i}\right)^{\perp} \\
\left(C_{i} D_{i}\right)^{+}
\end{array}\right] .
$$

Note that $K_{i}$ is nonsingular and its inverse matrix is

$$
K_{i}^{-1}=\left[\left(I-D_{i}\left(C_{i} D_{i}\right)^{+} C_{i}\right)\left(D_{i}^{\perp}\right)^{+} \quad D_{i}\right] \triangleq\left[\hat{K}_{i} \quad D_{i}\right] .
$$

By using the state and output transformation

$$
\bar{x}=\left[\begin{array}{cc}
\bar{x}_{1}^{T} & \bar{x}_{2}^{T}
\end{array}\right]^{T}=K_{\sigma(t)} x, \quad \bar{y}=\left[\begin{array}{cc}
\bar{y}_{1}^{T} & \bar{y}_{2}^{T}
\end{array}\right]^{T}=U_{\sigma(t)} y,
$$

then the switched system (1) becomes in the new coordinates

$$
\begin{aligned}
& \dot{\bar{x}}_{1}=A_{\sigma(t), 1} \bar{x}_{1}+A_{\sigma(t), 2} \bar{x}_{2}+B_{\sigma(t), 1} u+f_{\sigma(t), 1}(t, x), \\
& \dot{\bar{x}}_{2}=A_{\sigma(t), 3} \bar{x}_{1}+A_{\sigma(t), 4} \bar{x}_{2}+B_{\sigma(t), 2} u+w+f_{\sigma(t), 2}(t, x), \\
& \bar{y}_{1}=\bar{C}_{\sigma(t)} \bar{x}_{1}, \quad \bar{y}_{2}=\bar{x}_{2},
\end{aligned}
$$

where

$K_{\sigma(t)} A_{\sigma(t)} K_{\sigma(t)}^{-1}=\left[\begin{array}{ll}A_{\sigma(t), 1} & A_{\sigma(t), 2} \\ A_{\sigma(t), 3} & A_{\sigma(t), 4}\end{array}\right], K_{\sigma(t)} B_{\sigma(t)}=\left[\begin{array}{c}B_{\sigma(t), 1} \\ B_{\sigma(t), 2}\end{array}\right], \bar{x}_{1}=D_{\sigma(t)}^{\perp} x, \bar{x}_{2}=\left(C_{\sigma(t)} D_{\sigma(t)}\right)^{+} C_{\sigma(t)} x$, $\bar{y}_{1}=\left(C_{\sigma(t)} D_{\sigma(t)}\right)^{\perp} y, \bar{y}_{2}=\left(C_{\sigma(t)} D_{\sigma(t)}\right)^{+} y, f_{\sigma(t), 1}(t, x)=D_{\sigma(t)}^{\perp} f_{\sigma(t)}(t, x), f_{\sigma(t), 2}(t, x)=\check{K}_{\sigma(t)} f_{\sigma(t)}(t, x)$, $\bar{C}_{\sigma(t)}=\left(C_{\sigma(t)} D_{\sigma(t)}\right)^{\perp} C_{\sigma(t)}\left(D_{i}^{\perp}\right)^{\dagger}$. At switching instants $t_{k}$, from [16], the state of system (26) is governed by

$$
\bar{x}\left(t_{k}\right)=K_{\delta\left(t_{k}\right)} x\left(t_{k}^{-}\right)=K_{\sigma\left(t_{k}\right)} K_{\sigma\left(t_{k-1}\right)}^{-1} \bar{x}\left(t_{k}^{-}\right), k=1,2, \ldots
$$

According to (26), we only need to design observers for $\bar{x}_{1}$, because $\bar{x}_{2}$ can be directly obtained by $\bar{y}_{2}$. Furthermore, the dynamics (26) of $\bar{x}_{1}$ do not contain the unknown input $w$.

Consider the following subsystem relevant to $\bar{x}_{1}$ in (26)

$$
\begin{aligned}
& \dot{\bar{x}}_{1}=A_{\sigma(t), 1} \bar{x}_{1}+A_{\sigma(t), 2} \bar{x}_{2}+B_{\sigma(t), 1} u+f_{\sigma(t), 1}(t, x), \\
& \bar{y}_{1}=\bar{C}_{\sigma(t)} \bar{x}_{1}, \\
& \bar{x}_{1}\left(t_{k}\right)=D_{\sigma\left(t_{k}\right)}^{\perp} x\left(t_{k}^{-}\right), k=1,2, \ldots
\end{aligned}
$$

the proposed observer for system (28) has the form

$$
\begin{gathered}
\dot{\overline{\hat{x}}}_{1}=A_{\sigma(t), 1} \hat{\bar{x}}_{1}+A_{\sigma(t), 2} \bar{y}_{2}+B_{\sigma(t), 1} u+H_{\sigma}(t)\left(\bar{y}_{1}-\bar{C}_{\sigma(t)} \hat{\bar{x}}_{1}\right)+f_{\sigma(t), 1}(t, \hat{x}), \\
\hat{\bar{x}}_{1}\left(t_{k}\right)=D_{\sigma\left(t_{k}\right)}^{\perp} \hat{x}\left(t_{k}^{-}\right)=D_{\sigma\left(t_{k}\right)}^{\perp} \hat{K}_{\sigma\left(t_{k-1}\right)}\left[\begin{array}{c}
\hat{\bar{x}}_{1}\left(t_{k}^{-}\right) \\
\bar{y}_{2}\left(t_{k}^{-}\right)
\end{array}\right] k=1,2, \ldots \\
9
\end{gathered}
$$


where $H_{\sigma}(t)$ are the observer gains to be designed.

Define $e_{1}=\bar{x}_{1}-\hat{\bar{x}}_{1}$, we can obtain the following error system

$$
\left\{\begin{array}{l}
\dot{e}_{1}=\left(A_{\sigma(t), 1}-H_{\sigma(t)} \bar{C}_{\sigma(t)}\right) e_{1}+\Delta f_{\sigma(t), 1}(t, x, \hat{x}), \\
e_{1}\left(t_{k}\right)=D_{\sigma\left(t_{k}\right)}^{\perp} e\left(t_{k}^{-}\right)=D_{\sigma\left(t_{k}\right)}^{\perp} \hat{K}_{\sigma\left(t_{k-1}\right)} e_{1}\left(t_{k}^{-}\right), k=1,2, \ldots
\end{array}\right.
$$

where $\Delta f_{\sigma(t), 1}(t, x, \hat{x})=f_{\sigma(t), 1}(t, x)-f_{\sigma(t), 1}(t, \hat{x})$. From Assumption 1, one has

$$
\left\|\Delta f_{\sigma(t), 1}(t, x, \hat{x})\right\| \leq\left\|D_{\sigma(t)}^{\perp}\right\|\left\|F_{\sigma(t)} \hat{K}_{\sigma(t)} e_{1}\right\|
$$

In this section, similar to the full-order observer design, two classes of switched systems are considered. One is that all error subsystems are unstable, which implies that there is no restriction of the strong detectability condition for system (1) compared with [26, 27]; another case is that all error subsystems are stable.

Case c: all error subsystems are unstable.

Theorem 3.3. Consider switched system (1) satisfying Assumptions 1-2 and the time sequence $t_{k} \in$ $\Delta\left(\delta_{1}, \delta_{2}\right)$. For given $\eta_{1}>0,0<\mu_{1}<1, \alpha_{i}>0$ and $\rho_{i}>1$, if there exist $P_{i 1}>0, P_{i 2}>0, X_{i}>0, Y_{i}$, $\epsilon_{\text {imn }}>0, i, j \in M, i \neq j, m, n=1,2$, such that

$$
\begin{aligned}
& {\left[\begin{array}{ccc}
\Xi_{i m n} & P_{i m}-X_{i}-\alpha_{i} \bar{C}_{i}^{T} Y_{i}^{T} & P_{i m} \\
* & -\alpha_{i}\left(X_{i}+X_{i}^{T}\right) & 0 \\
0 & 0 & -\epsilon_{i m n} I
\end{array}\right]<0} \\
& {\left[\begin{array}{cc}
-\mu_{1} P_{j 1} & \left(D_{i}^{\perp} \hat{K}_{j}\right)^{T} P_{i 2} \\
* & -\rho_{i} P_{i 2}
\end{array}\right]<0,} \\
& \ln \mu_{1}+\eta_{1} \delta_{2}<0
\end{aligned}
$$

where

$\Xi_{i m n}=\theta_{i} P_{i m}+P_{i m} A_{i, 1}+A_{i, 1}^{T} P_{i m}-Y_{i} \bar{C}_{i}-\bar{C}_{i}^{T} Y_{i}^{T}+\frac{1}{\delta_{n}}\left(P_{i 1}-P_{i 2}\right)-\eta_{1} P_{i m}+\epsilon_{i m n}\left(F_{i} \hat{K}_{i}\right)^{T} F_{i} \hat{K}_{i}$ with $\theta_{i}=\frac{\ln \rho_{i}}{\delta_{1}}$, then the switched observer error dynamic system (30) is asymptotically stable. The observer gains are given by

$$
H_{i}=X_{i}{ }^{1} Y_{i}, i, \in M
$$

Proof: Suppose $\sigma(t)=i, t \in\left[t_{k}, t_{k+1}\right)$. Let $e_{1}(t)$ be a solution of system (30) and consider the Lyapunov function $V_{1 i}\left(e_{1}\right)=\psi(t) e_{1}^{T} P_{i}(t) e_{1}, i \in M$, denote $V_{1 i}(t)=V_{1 i}\left(e_{1}(t)\right)$. By pre- and post multiplying both sides of (32) with $\left[\begin{array}{ccc}I & -\left(H_{i} \bar{C}_{i}\right)^{T} & 0 \\ 0 & 0 & I\end{array}\right]$ and $\left[\begin{array}{ccc}I & -\left(H_{i} \bar{C}_{i}\right)^{T} & 0 \\ 0 & 0 & I\end{array}\right]^{T}$, one has

$$
\Psi_{i m n}=\left[\begin{array}{cc}
\Lambda_{i m n}-\eta_{1} P_{i m} & P_{i m} \\
* & -\epsilon_{i m n} I
\end{array}\right]<0
$$

where $\Lambda_{i m n}=\theta_{i} P_{i m}+H e\left\{P_{i m}\left(A_{i, 1}-H_{i} \bar{C}_{i}\right\}+\frac{1}{\delta_{n}}\left(P_{i 1}-P_{i 2}\right)+\epsilon_{i m n} F_{i}^{T} \hat{K}_{i}^{T} F_{i} \hat{K}_{i}\right.$. Similar to the proof of Theorem 3.1, we obtain that

$$
\dot{V}_{1 i}(t)-\eta_{1} V_{1 i}(t) \leq \psi(t)\left\{\kappa(t) \nu(t) \Pi_{i}^{11}+\kappa(t) \tilde{\nu}(t) \Pi_{i}^{12}+\tilde{\kappa}(t) \nu(t) \Pi_{i}^{21}+\tilde{\kappa}(t) \tilde{\nu}(t) \Pi_{i}^{22}\right\},
$$


where $\Pi_{i}^{m n}=\left[\begin{array}{cc}e_{1}^{T} & \Delta f_{i, 1}^{T}\end{array}\right] \Psi_{i m n}\left[\begin{array}{c}e_{1} \\ \Delta f_{i, 1}\end{array}\right]$. Combining (35) with (36), it follows that

$$
V_{1 i}(t) \leq V_{1 i}\left(t_{k}\right) \exp \left(\eta_{1}\left(t-t_{k}\right)\right), t \in\left[t_{k}, t_{k+1}\right)
$$

From (33), then

$$
\begin{aligned}
V_{1 i}\left(t_{k}\right) & =\psi\left(t_{k}\right) e_{1}^{T}\left(t_{k}\right) P_{i}\left(t_{k}\right) e_{1}\left(t_{k}\right) \\
& =\rho_{i}^{-1} e_{1}^{T}\left(t_{k}^{-}\right)\left(D_{i}^{\perp} \hat{K}_{j}\right)^{T} P_{i 2}\left(D_{i}^{\perp} \hat{K}_{j}\right) \\
& \leq \mu_{1} e^{T}\left(t_{k}^{-}\right) P_{j 1} e\left(t_{k}^{-}\right) \\
& =\mu_{1} V_{1 j}\left(t_{k}^{-}\right) .
\end{aligned}
$$

According to the condition (34), using the similar method in Theorem 3.1, we have $e_{1}(t) \rightarrow 0$ when $t \rightarrow+\infty$. The proof is completed.

Case d: all error subsystems are stable.

Theorem 3.4. Consider the switched system (1) satisfying Assumptions 1-2 and the time sequence $t_{k} \in \Delta\left(\delta_{1}, \delta_{2}\right)$. For given $\eta_{1}>0, \mu_{1}>0, \alpha_{i}>0$ and $\rho_{i}>1$, if there exist $P_{i 1}>0, P_{i 2}>0, X_{i}>0, Y_{i}$, $\epsilon_{\text {imn }}>0, i, j, l \in M, i \neq j, m, n=1,2$, such that (33) and the following inequalities hold:

$$
\begin{aligned}
& {\left[\begin{array}{ccc}
\Xi_{i m n} & P_{i m}-X_{i}-\alpha_{i} \bar{C}_{i}^{T} Y_{i}^{T} & P_{i m} \\
* & -\alpha_{i}\left(X_{i}+X_{i}^{T}\right) & 0 \\
* & 0 & -\epsilon_{i m n} I
\end{array}\right]<0} \\
& \ln u_{1}-\eta_{1} \delta_{1}<0
\end{aligned}
$$

where

$\Xi_{i m n}=\theta_{i} P_{i m}+P_{i m} A_{i, 1}+A_{i, 1}^{T} P_{i m}-Y_{i} \bar{C}_{i}-\bar{C}_{i}^{T} Y_{i}^{T}+\frac{1}{\delta_{n}}\left(P_{i 1}-P_{i 2}\right)+\eta_{1} P_{i m}+\epsilon_{i m n} F_{i}^{T} \hat{K}_{i}{ }^{T} F_{i} \hat{K}_{i}$ with $\theta_{i}=\frac{\ln \rho_{i}}{\delta_{1}}$, then the switched observer error dynamic system (30) is asymptotically stable. The observer gains are given by

$$
H_{i}=X_{i}^{-1} Y_{i}, i \notin M \text {. }
$$

Proof: The proof can be easily derived by the methodology given earlier. So we omit it here.

Remark 3.4. From (25), we have $\hat{x}(t)=K_{\sigma(t)}^{-1} \hat{\bar{x}}=K_{\sigma(t)}^{-1}\left[\begin{array}{c}\hat{\bar{x}}_{1} \\ \bar{y}_{2}\end{array}\right]$. Define $e=x-\hat{x}$, then $e(t)=$ $K_{\sigma(t)}^{-1}\left[\begin{array}{c}e_{1} \\ 0\end{array}\right]$. According to Theorems 3.3 and 3.4, $e_{1}(t)$ asymptotically converges to zero, it follows that $e(t)$ asymptotically converges to zero, which means the system states in (1) can be estimated effectively by the proposed reduced-order observers.

Remark 3.5. In [26-28], the observer design is based on the assumption that the matrix triplets $\left(A_{i}, C_{i}, D_{i}\right)$ have negative real part. However, in the reduced order design of our paper, this assumption is removed, which means that the observer can also be designed when none of the matrix pairs $\left(A_{i, 1}, \bar{C}_{i}\right)$ is required to be detectable, i.e., all error subsystems are unstable. This can be verified in following Example. 
Remark 3.6. When not all error subsystems are stable, the key point for designing full-order and reduced-order observers is how to ensure the stability of the whole error dynamic systems. In this case, according to the above four theorems, for $\sigma(t)=i, t \in\left[t_{k}, t_{k+1}\right), \sigma(t)=j, t \in\left[t_{k-1}, t_{k}\right)$, we have $\dot{V}_{i}(t)<$ $\eta_{i} V_{i}(t)$ and $V_{i}\left(t_{k}\right) \leq \mu V_{j}\left(t_{k}^{-}\right)$, where $\eta_{i}$ can be positive or negative. Choose $\eta_{\sigma\left(t_{k}\right)} \leq \eta, k=0,1,2, \ldots$, for $t \in\left[t_{k}, t_{k+1}\right)$, then

$$
\begin{aligned}
V_{\sigma(t)}(t) & \leq \mu^{N_{\delta}\left(t, t_{0}\right)} V_{\sigma\left(t_{0}\right)}\left(t_{0}\right) \exp \left[\eta_{\sigma\left(t_{1}\right)}\left(t_{1}-t_{0}\right)+\eta_{\sigma\left(t_{2}\right)}\left(t-t_{1}\right)+\cdots+\eta_{\sigma\left(t_{k+1}\right)}\left(t-t_{k}\right)\right] \\
& \leq V_{\sigma\left(t_{0}\right)}\left(t_{0}\right) \exp \left[N_{\delta}\left(t, t_{0}\right) \ln \mu+\eta\left(t-t_{0}\right)\right]
\end{aligned}
$$

where $N_{\delta}(T, t)$ denotes the number of impulsive times of the impulsive sequence $\delta$ on the interval $(t, T)$. Moreover, the average impulsive interval of the impulsive sequence $\delta=\left\{t_{1}, t_{2}, \ldots\right\}$ is equal to $T_{a}$ if there exist positive integer $N_{0}$ and positive number $T_{a}$, such that

$$
\frac{T-t}{T_{a}}-N_{0} \leq N_{\delta}(T, t) \leq \frac{T-t}{T_{a}}+N_{0}, \forall T \geq t \geq 0 .
$$

Based on this definition, if $\mu \geq 1$, then

$$
\left(\frac{t-t_{0}}{T_{a}}-N_{0}\right) \ln \mu \leq N_{\delta}\left(t, t_{0}\right) \ln \mu \leq\left(\frac{t-t_{0}}{T_{a}}+N_{0}\right) \ln \mu, \forall t \geq t_{0},
$$

which implies

$$
V_{\sigma(t)}(t) \leq V_{\sigma\left(t_{0}\right)}\left(t_{0}\right) \exp \left[\left(\frac{t-t_{0}}{T_{a}}+N_{0}\right) \ln \mu+\eta\left(t-t_{0}\right)\right]=\mu^{N_{0}} V_{\sigma\left(t_{0}\right)}\left(t_{0}\right) \exp \left[\left(\frac{\ln \mu}{T_{a}}+\eta\right)\left(t-t_{0}\right)\right] .
$$

If $0<\mu<1$, then we have

$$
V_{\sigma(t)}(t) \leq V_{\sigma\left(t_{0}\right)}\left(t_{0}\right) \exp \left[\left(\frac{t-t_{0}}{T_{a}}-N_{0}\right) \ln \mu+\eta\left(t-t_{0}\right)\right]=\mu^{-N_{0}} V_{\sigma\left(t_{0}\right)}\left(t_{0}\right) \exp \left[\left(\frac{\ln \mu}{T_{a}}+\eta\right)\left(t-t_{0}\right)\right] .
$$

Let $\bar{\mu}=\max \left\{\mu^{-N_{0}}, \mu^{N_{0}}\right\}$, it is obtained that

$$
V_{\sigma(t)}(t) \leq \bar{\mu} V_{\sigma\left(t_{0}\right)}\left(t_{0}\right) \exp \left[\left(\frac{\ln \mu}{T_{a}}+\eta\right)\left(t-t_{0}\right)\right] .
$$

Thus, from $\min \left\{\lambda\left(P_{i l}\right)\right\}\|e(t)\|^{2} \leq \rho_{i} V_{i}(t) \leq \rho_{i} \max \left\{\lambda\left(P_{i l}\right)\right\}\|e(t)\|^{2}$, it follows that

$$
\|\vec{e}(t)\|^{2} \leq \frac{\max \left\{\rho_{i}\right\} \bar{\mu}}{\min \left\{\lambda\left(P_{i l}\right)\right\}} V_{\sigma\left(t_{0}\right)}\left(t_{0}\right) \exp \left[\left(\frac{\ln \mu}{T_{a}}+\eta\right)\left(t-t_{0}\right)\right]
$$

where $i \in M$ and $l=1,2, \lambda\left(P_{i l}\right)$ is the eigenvalue of matrix $P_{i l}$. If $\frac{\ln \mu}{T_{a}}+\eta<0$, the error dynamic system is asymptotically stable. Therefore, when some error subsystems are stable and some are unstable, the full-order and reduced-order observer can also be constructed by designing the suitable impulsive switching rule that satisfies $\frac{\ln \mu}{T_{a}}+\eta<0$.

\section{Examples}

In this section, two examples are studied to show the effective of the proposed hybrid observers for switched nonlinear systems with unknown inputs. 
Example 1 Consider the following two subsystems:

$$
\begin{aligned}
& A_{1}=\left[\begin{array}{ccccc}
-1 & 0 & -1 & 1 & 1 \\
1 & -1 & 1 & -2 & -1 \\
1 & 1 & -1 & -1 & -0.1 \\
1 & -1 & 0 & -1 & 1 \\
1 & 1 & 3 & 1 & -1
\end{array}\right], A_{2}=\left[\begin{array}{ccccc}
-1 & 0 & 0 & 1 & 1 \\
1 & -1 & 1 & 2 & 1 \\
1 & 1 & -1 & 1 & 1 \\
1 & 0 & -1 & -1 & 0 \\
1 & -1 & 0 & -1 & -1
\end{array}\right], D_{1}=\left[\begin{array}{cc}
1 & 1 \\
1 & 1 \\
0 & 1 \\
0 & -1 \\
0 & 1
\end{array}\right], D_{2}=\left[\begin{array}{cc}
0 & 0 \\
0 & 1 \\
1 & 1 \\
0 & 0 \\
0 & 1
\end{array}\right], \\
& C_{1}=\left[\begin{array}{ccccc}
-1 & 0 & 0 & 1 & 0 \\
0 & 0 & 1 & 1 & 0 \\
0 & 1 & 0 & 0 & -1
\end{array}\right], C_{2}=\left[\begin{array}{ccccc}
0 & 0 & 0 & 1 & 0 \\
0 & 1 & 1 & 0 & 0 \\
-1 & 0 & 1 & 1 & 0
\end{array}\right], B_{1}=B_{2}=0,
\end{aligned}
$$

$f_{1}(t, x)=\left(\tanh \left(x_{1}\right), \tanh \left(x_{2}\right), \tanh \left(x_{3}\right), \tanh \left(x_{4}\right), \tanh \left(x_{5}\right)\right)^{T}, f_{2}(t, x)=\left(\sin \left(x_{1}\right), \sin \left(x_{2}\right), \sin \left(x_{3}\right), \sin \left(x_{4}\right)\right.$, $\left.\sin \left(x_{5}\right)\right)^{T}$. It can be verified that $\operatorname{rank}\left(C_{i} D_{i}\right)=\operatorname{rank}\left(D_{i}\right)=2, i=1,2$, which satisfies Assumption 2 . Let $\delta_{1}=0.02, \delta_{2}=0.04$. The switching and impulsive signal are shown in Figure 1.

Case a: When all error subsystems are not stable, an impulsive full-order observer is designed. Accord-

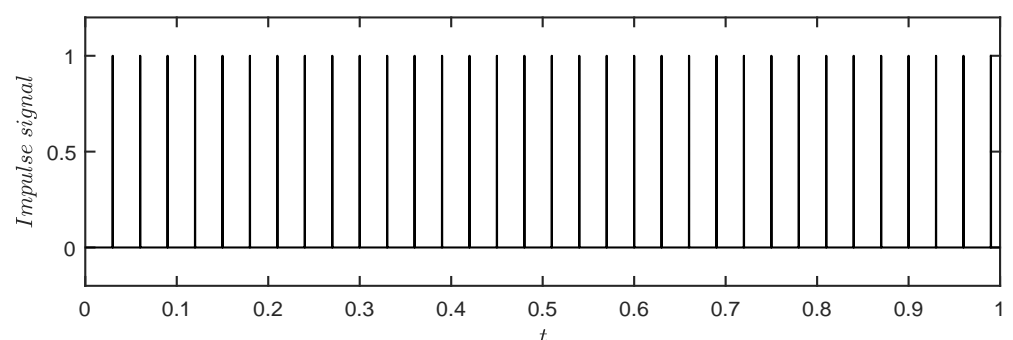

(a)

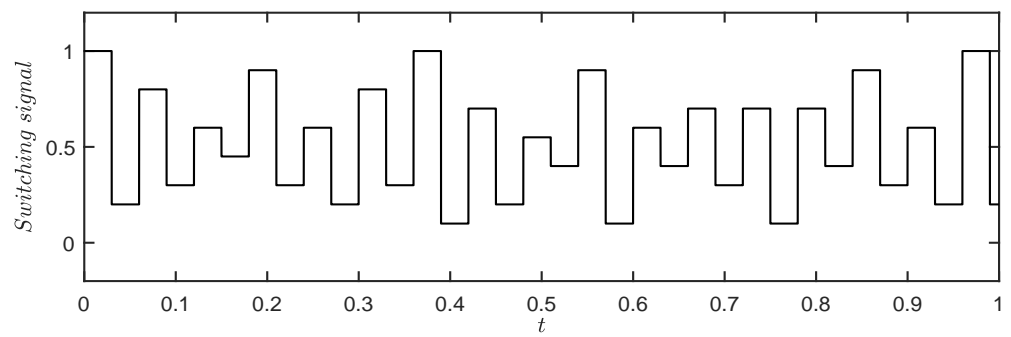

(b)

Figure 1: (a) Impulse signal (b) Switching signal.

ing to Algorithm for Theorem 3.1, choose $\rho_{1}=\rho_{2}=1.01, \eta=1, \mu=0.39$, we have

$$
L_{1}=\left[\begin{array}{ccc}
-0.6662 & 0.3329 & -0.0005 \\
0.2955 & -0.2955 & 0.2045 \\
-0.3329 & 0.6662 & -0.0005 \\
0.3333 & 0.3333 & -0.0000 \\
0.2946 & -0.2946 & -0.7946
\end{array}\right], L_{2}=\left[\begin{array}{ccc}
0.5680 & 0.1361 & -0.5680 \\
0.4311 & 0.8621 & -0.4311 \\
-0.4320 & 0.1361 & 0.4320 \\
1.0000 & -0.0000 & -0.0000 \\
0.2950 & 0.59000 & -0.2950
\end{array}\right] \text {, }
$$




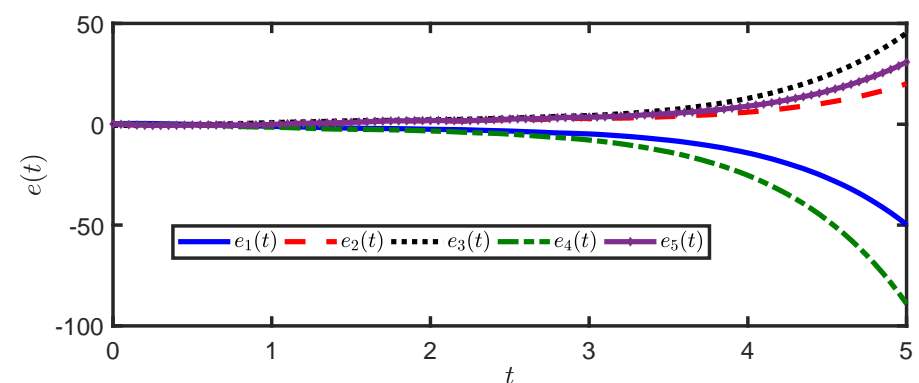

(a)

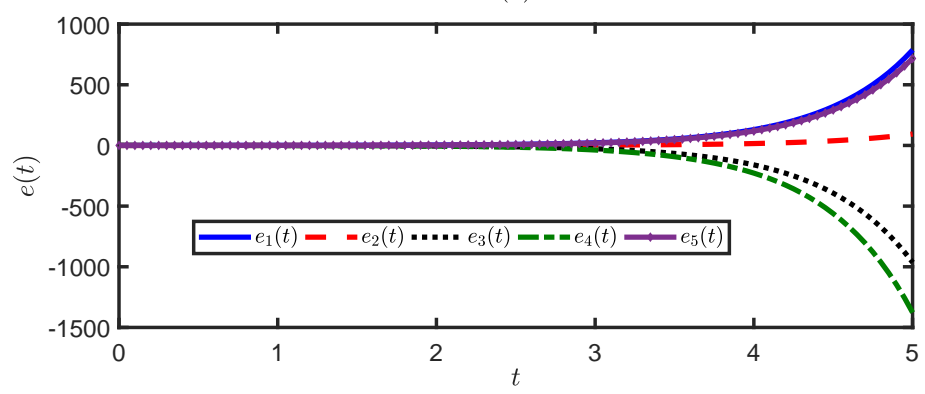

(b)

Figure 2: Trajectories of the error state $e$ of subsystems. (a) error subsystem 1. (b) error subsystem 2

$$
N_{1}=\left[\begin{array}{ccccc}
0.5000 & -0.5000 & 0.1265 & 0.6265 & 0.5000 \\
-0.5000 & 0.5000 & -1.3332 & -0.8332 & 0.5000 \\
-0.5000 & 0.5000 & -0.2159 & -0.7159 & -0.5000 \\
0.5000 & -0.5000 & -0.6898 & -0.1898 & 0.5000 \\
-0.5000 & 0.5000 & -1.1064 & -0.6064 & 0.5000
\end{array}\right], N_{2}=\left[\begin{array}{ccccc}
1 & 0 & 0 & 0.9114 & 0 \\
-1 & 0 & 0 & -0.4091 & 0 \\
1 & 0 & 0 & -0.0867 & 0 \\
0 & 0 & 0 & -0.7861 & 0 \\
-1 & -1 & 0 & -1.2242 & 1
\end{array}\right] .
$$

It should be noted that each subsystem of switched error system is unstable, since $\operatorname{eig}\left(N_{1} A_{1}\right)=\{1.2702+0.0000 i,-0.4588 \pm 1.6358 i,-0.0000 \pm 0.0000 i\}$, $\operatorname{eig}\left(N_{2} A_{2}\right)=\{0,-3.0000,1.7842,-1.0000,-0.0000\}$,

which means that the methods in $[21,24,25,27]$ can not be used to design the observer for the studied switched system.

Case b: When all error subsystems are stable. Choose $\eta=4, \mu=1.01$, solving inequalities in Theorem 3.2, the impulsiye gain matrices are given by

$$
L_{1}=\left[\begin{array}{ccc}
-0.3906 & 0.1321 & 0.0379 \\
0.2255 & -0.2386 & 0.1261 \\
-0.2107 & 0.4688 & -0.0367 \\
0.2101 & 0.1313 & 0.03755 \\
0.2252 & -0.2382 & -0.4735
\end{array}\right], L_{2}=\left[\begin{array}{ccc}
0.3140 & 0.0291 & -0.4127 \\
0.2877 & 0.5721 & -0.1878 \\
-0.2869 & 0.0282 & 0.1876 \\
0.6012 & 0.0009 & -0.0002 \\
0.0600 & 0.3876 & -0.0946
\end{array}\right]
$$

Next, the reduced-order switched observer is designed for above presented switched system. Following the time varying state transformation [16], we have 


$$
\begin{gathered}
A_{1,1}=\left[\begin{array}{ccc}
-2.3199 & -0.6217 & 2.1880 \\
1.3607 & 0.5309 & -1.1972 \\
1.2311 & 2.3774 & 0.2890
\end{array}\right], A_{2,1}=\left[\begin{array}{ccc}
-2 & -2 & -1.4142 \\
-0.0000 & -0.0000 & -0.0000 \\
-0.7071 & -2.8284 & -2
\end{array}\right] \\
\bar{C}_{1}=\left[\begin{array}{lll}
1 & 1 & 0
\end{array}\right], \bar{C}_{2}=\left[\begin{array}{lll}
0 & -1 & 0
\end{array}\right]
\end{gathered}
$$

Case c: when all the error systems are unstable. Let $\alpha_{1}=5.15, \alpha_{2}=5.05, \eta_{1}=5, \mu_{1}=0.2, \rho_{1}=\rho_{2}=$ 1.39. Solving LMIs in Theorem 3.3, we obtain observer gains for each subsystem:

$$
H_{1}=\left[\begin{array}{lll}
0.3730 & 0.0921 & 0.4289
\end{array}\right]^{T}, H_{2}=\left[\begin{array}{lll}
-0.0003 & 0.0000 & 0.0008
\end{array}\right]^{T} .
$$

It is verified that each subsystem of switched error system is unstable, since

$$
\operatorname{eig}\left(A_{11}-H_{1} \bar{C}_{1}\right)=\{-2.1112+0.0000 i, 0.0731 \pm 0.5565 i\}
$$

and

$$
\operatorname{eig}\left(A_{21}-H_{2} \bar{C}_{2}\right)=\{-3.0000,-1.0000,0.0001\}
$$

From [32], we can find the strong detectability of above two subsystems is not satisfied, which means the methods in [21, 25-29] can not be used to design observers for the studied switched system. Because in our paper, each error subsystem does not need to satisfy the strong detectability condition in the case of observer design.

Case d: when all the error systems are stable. Set $\alpha_{1}=\alpha_{2}=0.05, \eta_{1}=10.5, \mu_{1}=1.001$, solving LMIs in Theorem 3.4, the switched observer gains are given by

$$
H_{1}=\left[\begin{array}{lll}
14.9009 & 5.0992 & 15.1352
\end{array}\right]^{T}, H_{2}=\left[\begin{array}{lll}
20.0638 & -20 & 0.5292
\end{array}\right]^{T} .
$$

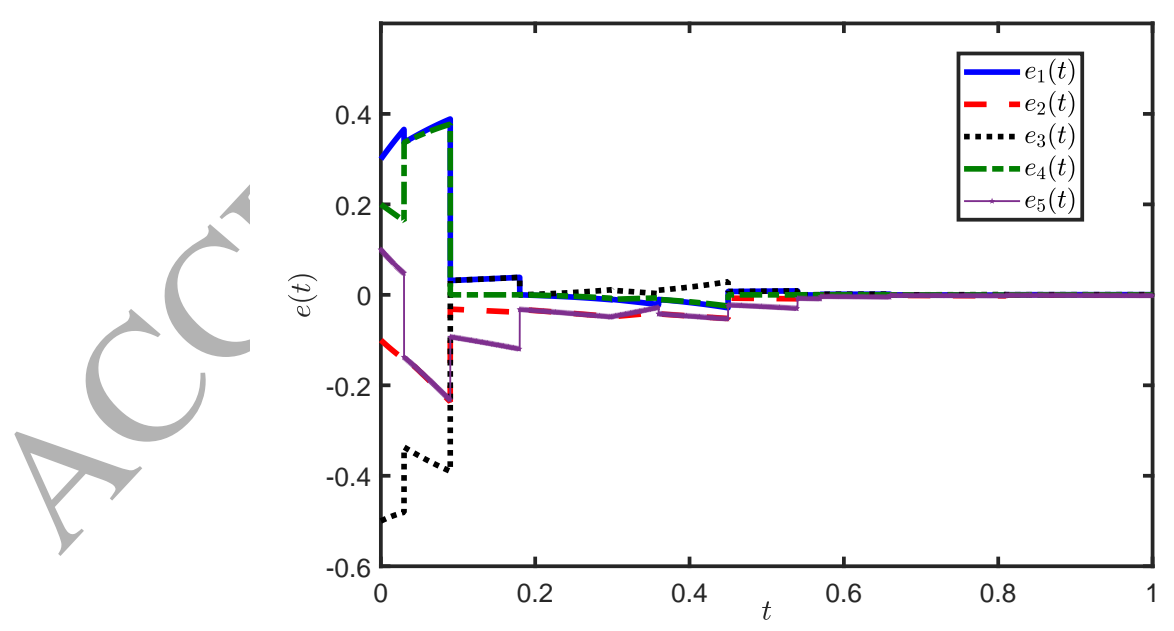

Figure 3: Trajectories of the switched error system with full-order impulsive observer.

Let $e\left(t_{0}\right)=(0.3,0.2,-0.5,0.2,0.1)^{T}$. From Figure 2, both subsystems of the switched error system are unstable in the case of full-order impulsive observer design. For the reduced-order switched observer design, two subsystem state responses perform similarly as it is shown in Figure 2 with state divergence. 


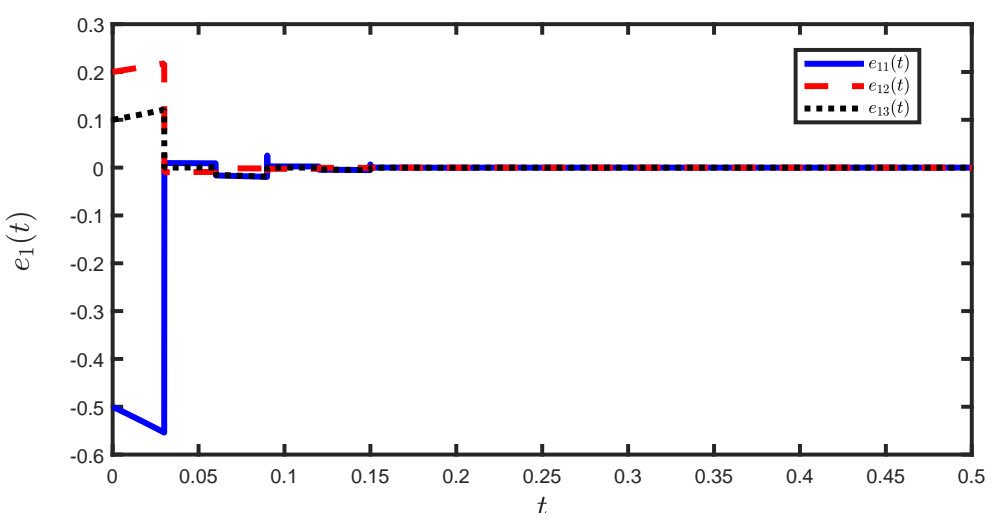

Figure 4: Trajectories of the switched error system with reduced-order switched observer.

Under the signal rule in Figure 2, the state trajectories of the whole dynamic of the observation error are presented in Figures 3 and 4. It can be clearly observed that the asymptotical stability of the switched error systems (6) and (30) have been achieved. Thus, the simulation results well show the effectiveness of the proposed full-order and reduced-order observers.

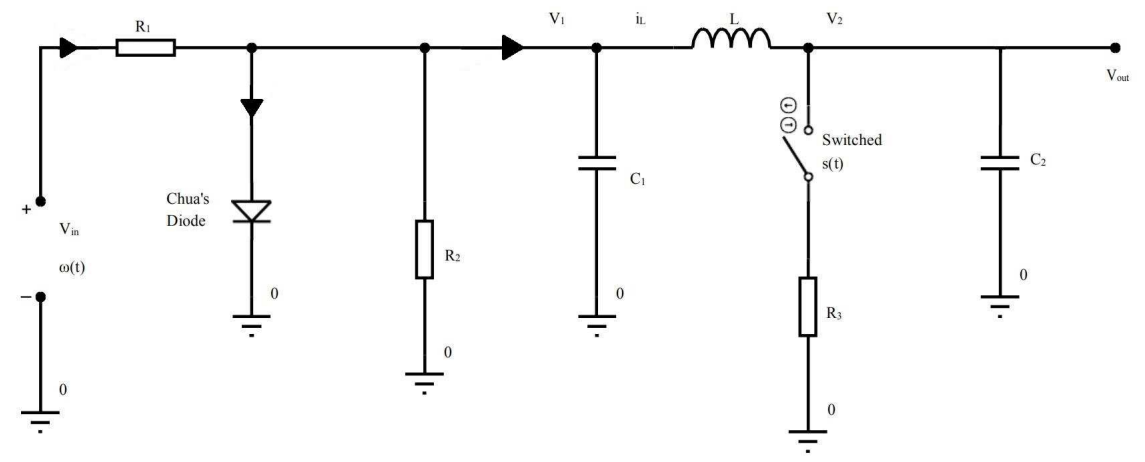

Figure 5: The electronic circuit.

Example 2 Consider a practical electronic circuit in Figure 5, where $C_{i}(i=1,2)$ are capacitors, $V_{i}$ are the corresponding voltages that can be regarded as the measurement outputs. $R_{i}(i=1,2,3)$ are resistors, $L$ is an inductor, $w(t)$ is unknown value of the voltage resource. The Chua's diode is a nonlinear resistor [29] that satisfies the following condition

$$
I=f(u)=\left\{\begin{array}{l}
-1.143 U-0.429, U \leq 1 \\
-0.714 U,-1<U \leq 1 \\
-1.143 U+0.429, U>1
\end{array}\right.
$$

where $U, I$ stand for the voltage and the current of the Chua's diode, respectively. Then the circuit 
system is written by

$$
\left\{\begin{array}{l}
\dot{i}_{L}(t)=\frac{1}{L} V_{1}(t)-\frac{1}{L} V_{2}(t), \\
\dot{V}_{1}(t)=-\frac{1}{C_{1}} i_{L}(t)-\frac{1}{C_{1}} f\left(V_{1}\right)-\frac{1}{C_{1} R_{2}} V_{1}(t)+\frac{1}{C_{1} R_{1}} w(t), \\
\dot{V}_{2}(t)=\frac{1}{C_{2}} i_{L}(t)-\frac{s(t)}{C_{2} R_{3}} V_{2}(t),
\end{array}\right.
$$

where $s(t)=\left\{\begin{array}{ll}1, & \text { switch on } \\ 0, & \text { switch of } f\end{array}\right.$ is used to describe the switching between the two modes of the electronic circuit. Let $x=\left[\begin{array}{lll}x_{1} & x_{2} & x_{3}\end{array}\right]^{T}=\left[\begin{array}{lll}i_{L}(t) & V_{1}(t) & V_{2}(t)\end{array}\right]^{T}$, then we have the system

$$
\left\{\begin{array}{l}
\dot{x}(t)=A_{i} x(t)+D_{i} w(t)+f_{i}(t, x(t)) \\
y(t)=C_{i} x(t), \quad i=1,2
\end{array}\right.
$$

where

$$
\begin{gathered}
A_{1}=\left[\begin{array}{ccc}
0 & \frac{1}{L} & -\frac{1}{L} \\
-\frac{1}{C_{1}} & -\frac{1}{C_{1} R_{2}} & 0 \\
\frac{1}{C_{2}} & 0 & -\frac{1}{C_{2} R_{3}}
\end{array}\right], A_{2}=\left[\begin{array}{ccc}
0 & \frac{1}{L} & -\frac{1}{L} \\
-\frac{1}{C_{1}} & -\frac{1}{C_{1} R_{2}} & 0 \\
\frac{1}{C_{2}} & 0 & 0
\end{array}\right], B_{1} \mp B_{2}=0, \\
C_{1}=C_{2}=\left[\begin{array}{lll}
0 & 1 & 0 \\
0 & 0 & 1
\end{array}\right], D_{1}=D_{2}=\left[\begin{array}{c}
0 \\
\frac{1}{C_{1} R_{1}} \\
0
\end{array}\right], f_{1}(t, x)=f_{2}(t, x)=\left[\begin{array}{c}
0 \\
-\frac{1}{C_{1}} f\left(x_{2}\right) \\
0
\end{array}\right] .
\end{gathered}
$$

Choose $L=1, C_{1}=2, C_{2}=1, R_{1}=1, R_{2}=0.5, R_{3}=1$, and $w(t)=2 \sin (5 t)$. Assumption 2 is satisfied, and then denote $\delta_{1}=0.5, \delta_{2}=1.5$. Based on Algorithm for the full-order observer design,
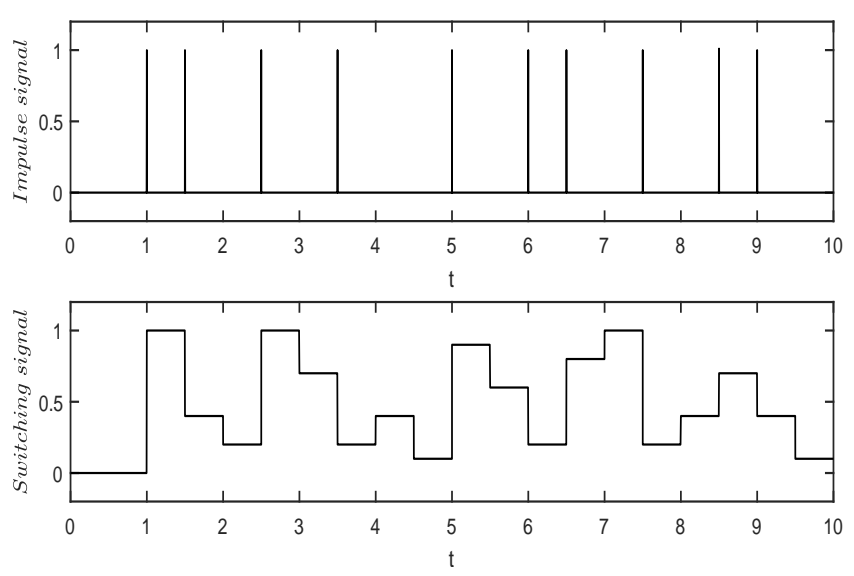

Figure 6: (a) Impulse signal (b) Switching signal.

$\eta=1.3, \mu=0.001, \rho_{1}=\rho_{2}=0.01$, then the condition $\ln \mu+\eta \delta_{2}=-4.9578<0$, we have

$$
L_{1}=\left[\begin{array}{cc}
-0.0184 & 0.0184 \\
3.3010 & 0.0000 \\
-0.000 & 3.3010
\end{array}\right], L_{2}=\left[\begin{array}{cc}
0.0007 & 35.1172 \\
3.3010 & -0.0000 \\
0.0000 & 3.3010
\end{array}\right], G_{1}=\left[\begin{array}{cc}
0 & 0.8454 \\
0 & -0.8887 \\
0 & 1.1980
\end{array}\right], G_{2}=\left[\begin{array}{cc}
0 & -1.7976 \\
0 & 1.2928 e-04 \\
0 & 0.9999
\end{array}\right] .
$$

For the reduced order observer design, according to the time varying state transformation,

$$
A_{1,1}=\left[\begin{array}{cc}
0 & 1 \\
-1 & -1
\end{array}\right], A_{2,1}=\left[\begin{array}{cc}
0 & 1 \\
-1 & 0
\end{array}\right], \bar{C}_{1}=\bar{C}_{2}=\left[\begin{array}{ll}
0 & 1
\end{array}\right]
$$


Choose $\alpha_{1}=5, \alpha_{2}=16, \eta_{1}=4, \mu_{1}=0.002, \rho_{1}=\rho_{2}=1$, the equality $\ln \mu_{1}+\eta_{1} \delta_{2}=-0.2146<0$ is satisfied, and then solving LMIs in Theorem 3.3, the observer gain matrices are given by

$$
H_{1}=\left[\begin{array}{c}
-0.0163 \\
0.2000
\end{array}\right], H_{2}=\left[\begin{array}{c}
-0.0000 \\
-0.0019
\end{array}\right] \text {. }
$$

In this example, the corresponding switched impulsive signal is presented in Figure 6. Under the initial condition $e\left(t_{0}\right)=(-0.2,0.5,-0.4)^{T}$, Figures 7 and 8 show that the switched error dynamic systems $(6)$ and (30) are asymptotically stable under the designed full-order and reduced-order observer respectively, which implies that both the full-order and reduced-order observers have good estimation performances.

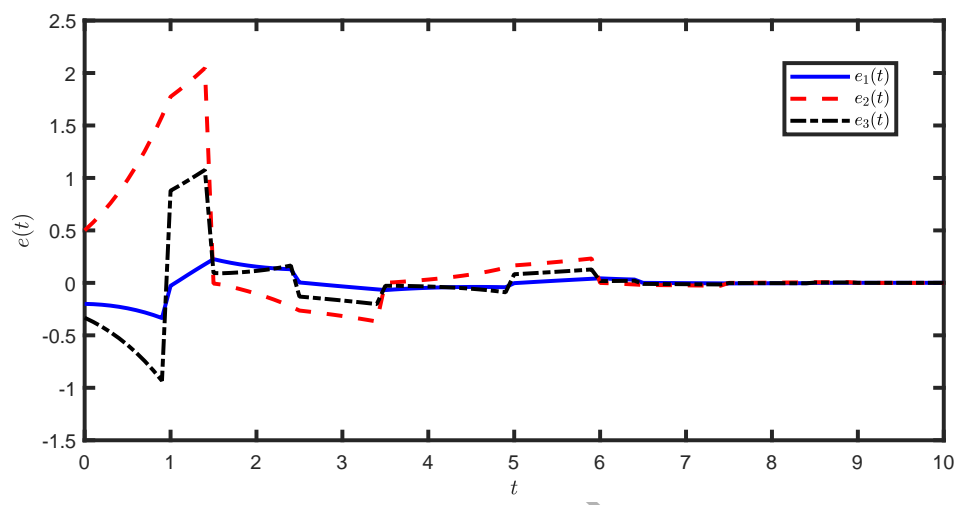

Figure 7: Trajectories of the switched error system with full-order impulsive observer.

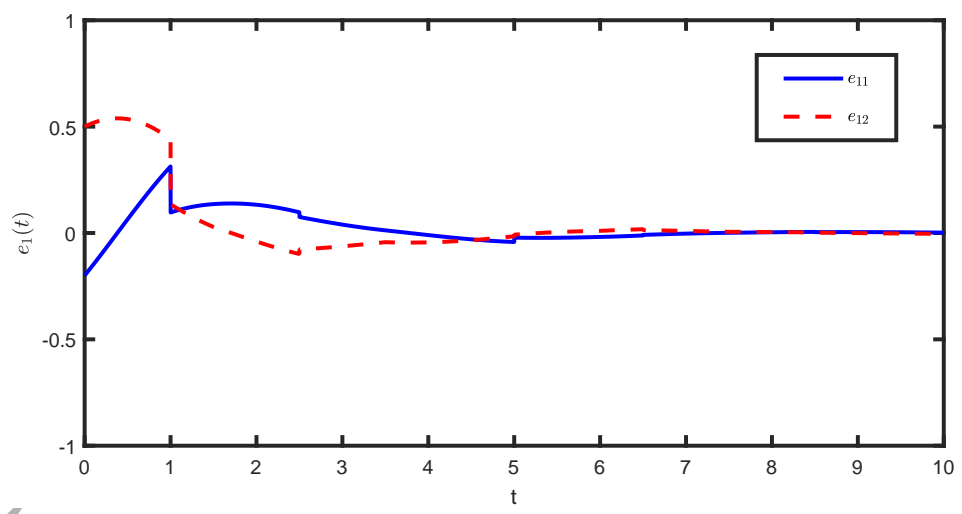

Figure 8: Trajectories of the switched error system with reduced-order switched observer.

\section{Conclusion}

This paper has investigated a full-order impulsive and reduced-order switched observer via dwell-time switchings without requiring the stability of each error subsystem of the switched systems. In spite of the instability of individual subsystems, the stability of the error switched system is achieved by confining the dwell time within an upper bounds. In addition, the multiple time-varying Lyapunov functions method is also used to analyze the stability analysis of a class of switched error systems with stable subsystems. 
Based on the estimated state information produced by the designed observer in this paper, a future research will focus on some practical control problems [33, 34]. Finally, the simulation results have shown the effectiveness of the proposed criteria.

\section{References}

\section{References}

[1] D. Liberzon, Switching in systems and control, Springer Science \& Business Media, 2012.

[2] G. Xie, D. Zheng, L. Wang, Controllability of switched linear systems, IEEE Transactions on Automatic Control 47 (8) (2002) 1401-1405.

[3] X. Zhao, L. Zhang, P. Shi, M. Liu, Stability and stabilization of switched linear systems with mode-dependent average dwell time, IEEE Transactions on Automatic Control 57 (7) (2012) 1809-1815.

[4] H. Yang, B. Jiang, J. Zhao, On finite-time stability of cyclic switched nonlinear systems, IEEE Transactions on Automatic Control 60 (8) (2015) 2201-2206.

[5] C. Briat, Dwell-time stability and stabilization conditions for linear positive impulsive and switched systems, Nonlinear Analysis: Hybrid Systems 24 (2017) 198-226.

[6] A. Tanwani, H. Shim, D. Liberzon, Observability for switched linear systems: Characterization and observer design, IEEE Transactions on Automatic Control 58 (4) (2013) 891-904.

[7] L. Zhou, J. She, S. Zhou, C. Li, Compensation for state-dependent nonlinearity in a modified repetitive control system, International Journal of Robust and Nonlinear Control 28 (1) (2018) 213-226.

[8] X. Zhao, H. Liu, J. Zhang, H. Li, Multiple-mode observer design for a class of switched linear systems, IEEE Transactions on Automation Science and Engineering 12 (1) (2015) 272-280.

[9] S. Yin, H. Gao, J. Qiu, O. Kaynak, Descriptor reduced-order sliding mode observers design for switched systems with sensor and actuator faults, Automatica 76 (2017) $282-292$.

[10] C. Edwards, S. K. Spurgeon, R. J. Patton, Sliding mode observers for fault detection and isolation, Automatica 36 (4) (2000) 541-553.

[11] M. Chen, W. Min, Unknown input observer based chaotic secure communication, Physics Letters A 372 (10) (2008) 1595-1600.

[12] Y. PARK, J. L. STEIN, Closed-loop, state and input observer for systems with unknown inputs, International Journal of Control 48 (3) (1988) 1121-1136.

[13] D. Koenig, B. Marx, D. Jacquet, Unknown input observers for switched nonlinear discrete time descriptor systems, IEEE Transactions on Automatic Control 53 (1) (2008) 373-379.

[14] J. Lin, Y. Shi, Z. Gao, J. Ding, Functional observer for switched discrete-time singular systems with time delays and unknown inputs, IET Control Theory \& Applications 9 (14) (2015) 2146-2156.

[15] L. Zhou, J. She, S. Zhou. Robust $H_{\infty}$ control of an observer-based repetitive-control system, Journal of the Franklin Institute 355 (12) (2018) 4952-4969.

[16] R. Ma, J. Fu,/T. Chai, Dwell-time-based observer design for unknown input switched linear systems without requiring strong detectability of subsystems, IEEE Transactions on Automatic Control 62 (8) (2017) 4215-4221.

[17] X. Li, P. Li, Q. G. Wang, Input/output-to-state stability of impulsive switched systems, Systems \& Control Letters 116 (2018) $1-7$.

[18] X. Li, D. W. Ho, J. Cao, Finite-time stability and settling-time estimation of nonlinear impulsive systems, Automatica 99 (2019) 361-368.

[19] T. Zhan, S. P. Ma, H. Chen, Impulsive stabilization of nonlinear singular switched systems with all unstable-mode subsystems, Applied Mathematics and Computation 344 (2019) 57-67.

[20] T. Raff, F. Allgower, Observers with impulsive dynamical behavior for linear and nonlinear continuous-time systems, in: Decision and Control, 2007 46th IEEE Conference on, IEEE, 2007, pp. 4287-4292.

[21] W. H. Chen, D. X. Li, X. Lu, Impulsive functional observers for linear systems, International Journal of Control, Automation and Systems 9 (5) (2011) 987. 
[22] M. Ayati, H. Khaloozadeh, X. Liu, Synchronizing chaotic systems with parametric uncertainty via a novel adaptive impulsive observer, Asian journal of Control 13 (6) (2011) 809-817.

[23] M. Ayati, H. Khaloozadeh, Designing a novel adaptive impulsive observer for nonlinear continuous systems using lmis, IEEE Transactions on Circuits and Systems I: Regular Papers 59 (1) (2012) 179-187.

[24] W. H. Chen, W. Yang, W. X. Zheng, Adaptive impulsive observers for nonlinear systems: Revisited, Automatica 61 (2015) 232-240.

[25] J. Li, R. Ma, G. M. Dimirovski, Adaptive impulsive observers for a class of switched nonlinear systems with unknown parameter, Asian Journal of Control 19 (3) (2017) 1153-1163.

[26] F. J. Bejarano, A. Pisano, Switched observers for switched linear systems with unknown inputs, IEEE Transactions on Automatic Control 56 (3) (2011) 681-686.

[27] G. J. Huang, W. H. Chen, A revisit to the design of switched observers for switched linear systems with unknown inputs, International Journal of Control, Automation and Systems 12 (5) (2014) 954-962.

[28] J. Yang, F. Zhu, X. Tan, Y. Wang, Robust full-order and reduced-order observers for a class of uncertain switched systems, Journal of Dynamic Systems, Measurement, and Control 138 (2) (2016) 021004.

[29] J. Zhang, F. Zhu, X. Zhao, F. Wang, Robust impulsive reset observers of a class of switched nonlinear systems with unknown inputs, Journal of the Franklin Institute 354 (7) (2017) 2924-2943.

[30] R. Sakthivel, T. Saravanakumar, Y. K. Ma, S. M. Anthoni, Finite-time resilient reliable sampled-data control for fuzzy systems with randomly occurring uncertainties, Fuzzy Sets and Systems $329(2017)$ 1-18.

[31] Y. Liu, J. H. Park, B. Z. Guo, Y. Shu, Further results on stabilization of chaotic systems based on fuzzy memory sampled-data control, IEEE Transactions on Fuzzy Systems 26 (2) (2018) 1040-1045.

[32] M. L. Hautus, Strong detectability and observers, Linear Algebra and its applications 50 (1983) 353-368.

[33] B. Zhang, Q. Han, X. Zhang, X. Yu, Sliding mode control with mixed current and delayed states for offshore steel jacket platforms, IEEE Transactions on Control Systems Technology 22 (5) (2014) 1769-1783.

[34] B. Zhang, Q. Han, X. Zhang, Recent advances in vibration control of offshore platforms, Nonlinear Dynamics 89 (2) (2017) $755-771$.

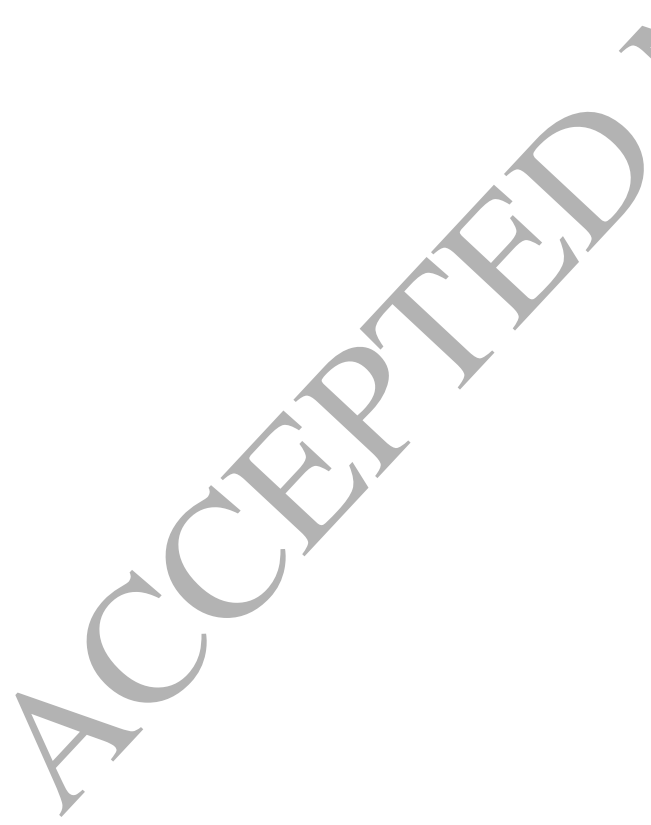

